\title{
Methods for Web-Spam Detection on web: Principles and Algorithms
}

\author{
Parminder Kaur \\ Shri Venkateshwara University, Gajraula, India \\ Available online at: www.isroset.org \\ Received: 10/Apr/2018, Revised: 15/Apr/2018, Accepted: 26/Apr/2018, Online: 30/Apr/ 2018
}

\begin{abstract}
A excess of big data applications are emerging which is being researched in the field of information technology which needs recognition of pattern and online classification of large dataset fetched from various forum working on online platform. The present research focuses on systematically analyzing and categorizing models that detect review spam. However, spamming is considered as critical issue in web mining. To handle the difficult queries, research is conducted on algorithm for data mining and knowledge discovery. I started with the introduction of web mining, web spam and process of mining Next, the study proceeds to assess them in terms of accuracy and results. Different detection techniques have different strengths and weaknesses and thus favor different detection contexts. The simulation output of our approach on different queries which shows effectiveness of our proposed framework. As the final part, we provide our conclusion and prospect.
\end{abstract}

Keywords: Web, Internet, Server, Spam, Detection Technique

\section{Introduction}

A web document is a document that can be is accessible through the facility of Internet or other network using various components of internet, by entering a URL address on the address bar that may contain variety of text, graphics, and hyperlinks to other web pages and files.

The paper is organized as follows, Section I contains the introduction of web spam, web search engine related to web content mining, Section II contain the related work of knowledge discovery in spam detection, Section III contain the some measures and comparisons of spam detection algorithms , Section IV contain the result and observation of proposed research work and Section V concludes research work with future directions.

\subsection{Define web spam (keywords and links)}

Spam is considered as a practice of sending unsolicited, uncalled emails to a large count of user. Unfortunately, it is becoming a regular practice of small size businesses and internet pro marketers trying to do their selling business of products or services. It is observed that every average computer have $20 \%$ to $30 \%$ spam email in their email box. As a result, this is a reason for large demand of anti-spam software in the market. On the web search engines, the major threats and challenges are related to Web spam and this has become the most challenging threat. There is term adversarial information retrieval, which shows the relationship between the search systems and those who try to manipulate it. 


\subsection{Web Search Engines}

Web search Engines are the tools for fetching the data or useful information on the World Wide Web. Generally, it is observed that popular pages can be view on the top few pages as the result of searching algorithm for the web users. Therefore, to prompt one of the sites rank at the first pages of the result of search engine, content providers use improper ways then this act as spam indexing or we can say to gain top most position rank in the result of the search engine, which is not actually feasible for the normal user. It is observed, that the process of manual spam detection is costly, slow and hard to automate. The success of search engine for better Understanding of all spamming techniques and dealing with these techniques is always the critical issue.

In the past two decades it is noticed that the database systems successful comes into existence. Vast amount of data and information are collected and then stored into variety of databases; now- adays Petabyte (1024 terabytes) is become very popular term in context to database. The main center of attention of many organizations searching needful information in such databases and pays more attention to turn a web mining is a main key unit of that type of information discovery. For designing patterns for web pages or creating meaningful forecasts algorithms based on web mining and its tools are required to design pages based on it. There are various application areas for such kind of technology is banking, marketing, finance, business, e-business, ecommerce, communication etc.

Web mining is a process used with different types of warehouses to mine different forms of information. However, to mine variety of data from various repositories, one can approach different methods, approaches, and algorithm as per the need. Indeed, it is always a challenges faced while dealing with different types of data. For the effective use of Web mining, it is necessary to study level of databases like RDB (Relational databases), ORD (Object-Relational databases), OOD (object-oriented databases), distribute data warehouses, centralized data warehouse, transaction databases, structure, unstructured and semi-structured repositories which is known as the World Wide Web, Latest databases i.e. text-based database, time-constraint database, spatial databases, multimedia databases and flat files in addition of databases. Usually, the web structure of data warehouses are designed in form of multidimensional web structure for the use of unified schema and it is considered as mass repository for heterogeneous and homogeneous environment.

\subsection{Data Mining as Process}

Data Mining is a one of the process which is used to develop programs based on computer program for analysis the raw data i systematic manner in respect of models, rules and regularities. To carry forward such kind of task one need some tools and techniques like figures, user friendly methods based on visualization, concepts of automata, simulated learning. Basically, the Data Mining is a process of iteration and partially automated who may need manual involvement in many important areas. $\mathrm{n}$

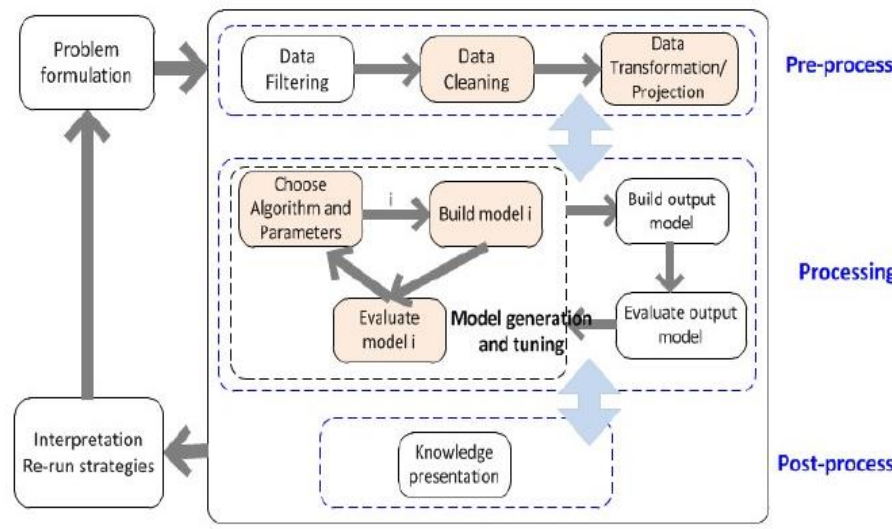

Figure 1.1 - Main steps of Data Mining Process

\section{Literature Review}

The literature survey has been done to discover or explore the former works carried out in the applicable areas such as:

- Content Mining 
- Intelligent Web Mining

- Knowledge discovery of spam detection

" Key practical aspects of training these models with large data sets are discussed, along with the role of GPU computing." (Witten et. al., 2017). It was observed that they are based on concepts, i.e. decision trees vs. artificial neural networks, and finally search the results for complex, high dimensional and non-linear data modeling. "The Group concluded, that the study discusses several important issues of data driven modeling with the selection and uncertainties. There approach is considered as simulated the real data case studies with resources assessment and natural hazards tasks.

"At end it was determined that the current challenges and future developments in statistical environmental data learning are discussed."(Leuenberger, 2016). "It was determined that experimental setup mainly focuses with the web structure retrieval nodes and hyperlinks as per to identify popular web page. "It was observed that web structure retrieval process of variant effect of periodic web pages in the field of educational domain which can be carried out with known optimal output strategies."

"On the bases of Financial Fraud Detection with an increase in financial accounting fraud in the current economic scenario experienced, financial accounting fraud detection (FAFD) is seems to be great importance for academic, research and industries. To search out the reasons for failure of auditing system in the organization implements procedures to detect financial accounting fraud which is also known as forensic accounting to identifying the accounting frauds. It was determined that it is complex to deal with the financial data for forensic accounting in order to provide various aids in this field the data mining methodology. The group concluded that there are various tool and techniques for accounting fraud detection and it may be provide strong foundation of future research in this field. They also suggested that findings of this review show that data mining techniques like logistic models, neural networks, Bayesian belief network, and decision trees have been applied most extensively to provide primary solutions to the problems inherent in the detection and classification of fraudulent."( Sharma et. Al., 2013).

"Search engines became a de facto place to start information acquisition on the Web. Though due to web spam phenomenon, search results are not always as good as desired. Moreover, spam evolves that makes the problem of providing high quality search even more challenging. Over the last decade research on adversarial information retrieval has gained a lot of interest both from academia and industry. In this paper we present a systematic review of web spam detection techniques with the focus on algorithms and under- lying principles. We categorize all existing algorithms into three categories based on the type of information they use: content-based methods, link-based methods, and methods based on nontraditional data such as user behavior, clicks, HTTP sessions. In turn, we perform a sub categorization of link-based category into five groups based on ideas and principles used: labels propagation, link pruning and reweighting, labels refinement, graph regularization, and featurebased. We also define the concept of web spam numerically and provide a brief survey on various spam forms. Finally, we summarize the observations and underlying principles applied for web spam detection.”(Hans, et. al.,2012)

"Data mining techniques and applications - A decade review. They review in order to determine how data mining techniques (DMT) and their applications have developed, during the past decade, the group concluded that data mining techniques and their applications and development, through a survey of literature and the classification of articles. They also explained the implementation of DMT, in respect to three basic areas: knowledge types, analysis types, and architecture types, integrated with their 
applications in different research domains. They discussed with the direction of any future developments in DMT methodologies and applications like DMT is a problem-oriented domain with expertise orientation and the development of applications in finding increasing applications implementation. The group also suggested that with the techniques and tools of DMT one can easy determined human behavior with different social science methodologies, such as psychology and cognitive science this can extent to better understanding with new future perspective." (Liao et. al., 2012).

"A task called topic anatomy, which conclude the main parts of a topic for not permanent and easily understand by the readers. The proposed topic anatomy model, called TSCAN with temporal block association matrix. It was determined that the required techniques focused on cutoff of cost and memory consumption at the time of dealing with databases. The group concludes that it is important to find new techniques for frequent item sets for both conventional database and temporal databases." (Chien et. al., 2012)

\section{Following listed algorithms are based on spam detection algorithms:}

\subsection{TrustRank}

TrustRank is considered as a strong theoretical relation with PageRank algorithm. This kind of algorithm separates reliable good pages from spam and to identify more and additional good pages in the link structure in order to find out good pages by using semi-automatically techniques. The perception behind TrustRank is that good pages automatically find out the bad pages. This process is started by selecting seeds. In the procedure of Seed selection the universal PageRank will be apply to the data set in order to obtain useful normal pages to identify extra pages. One more investigative towards this is to obtain high rank pages.

\subsection{PageRank}

It has been noticed that a high PageRank page will always point to another high PageRank page, thereby as a propagating trust. The outcome can be ranked in reverse parameter and the quality pages are selected from the L, which is consider like top pages as the good seed set because it is observed that trust streams from the good seed set only. For normalization, TrustRank distributes vector and implements Eq. 1, like to PageRank with less alteration:

$$
t^{*}=\alpha \cdot T \cdot t^{*}+(1-\alpha) \cdot d
$$

For Eq. (1), decay factor is a "a" it value is usually set at 0.85, where Transition Matrix is $\mathrm{T}$; and after normalization, distribution vector d. Similar to PageRank it is called an iterative algorithm, which is used to calculate the M iterations.

Decline with a 0.85 successively in iteration $M 1 / 4$ with 50 recapitulations and fix the value of $\mathrm{L} 1 / 43$ with $\mathrm{sp} 1 / 4 \mathrm{fFg}$ with $\mathrm{s} \grave{\mathrm{A}} 1 / 4 \mathrm{fD}$, for example $\mathrm{F}$ is a propagates trust to good pages $\mathrm{C}, \mathrm{B}$ and $\mathrm{A}$ and all of them are refer as a high PageRank values in $\mathrm{M}$ iteration, whereas pages $\mathrm{D}$ and $\mathrm{E}$ considered as a have small value for PageRank. Page $F$ is mentioned as supported page because its comes in the category of page of good value, and page D and $\mathrm{E}$ are devalued as being bad pages as compare to other pages.

\subsection{Derivatives of TrustRank Algorithms 3.3.1 ANTI-TRUSTRANK}

Anti-Trust Rank algorithm is transmitted in the opposite route or direction to trust rank algorithm, one factor is common in both algorithm is that they both follows the isolation principles in the same approximate. Anti-Trust Rank algorithm uses insource links of spam pages 


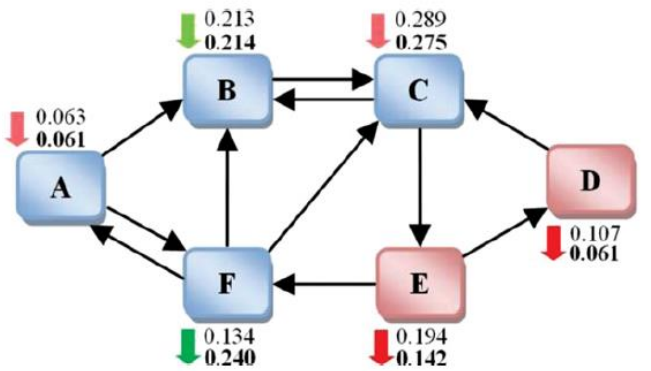

Figure 3.4: Simple Web Graph with PageRank and TrustRank

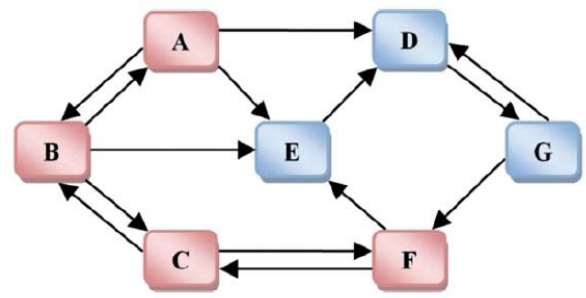

Figure 3.5: Good pages and Bad Pages in Simple web Graph

A Page is regarded as a spam page if given threshold value is less than the Anti-TrustRank score of the page. Figure shows, after assuming that Page A is known as a seed set of spam pages, and pages belongs to the category of Anti-Trust Rank would transfer to page $B$, and page B would transmit to page $\mathrm{F}$ and to page $\mathrm{C}$, but only if the calculated scores for such pages were greater than the defined value.

Initially, an algorithm of anti-trust rank estimates value for data set with the help of the PageRank algorithm and then it selects high Page Rank with a seed set on the spam page; usually it is found that the pages holding the value of high PageRank are targeted page as compared to the low rank page. It makes the task easier and faster to detect other spam pages that having high PageRank By using high PageRank spam seed set. Then, AntiTrustRank executes the PageRank algorithm with the help of transpose matrix spam in seed set which represents the web graph. It is observed at end, PageRank implement to find out the content from the non-reliable data, and arranged them in descending order. Spam Pages are the pages those mark as a higher threshhold value after calculation and estimation.

With the help of algorithm like Anti-Trust Rank algorithm one can estimate the untrust worthy pages data from the calculated report of seed set with the shortest path. In addition to this, we found that Anti-TrustRank calculated the average spam page rank was higher than the average spam page rank. When it is summarized, Anti-TrustRank has the advantage of giving high precision spam pages. The idea behind it is that with the starting in set of seed spam pages as high rank in the PageRank, would be assume, that perform recessive method in order to find out the spam pages amongst huge set of pages.

\subsection{TOPICAL TRUSTRANK}

The seed selection method of the Trust Rank algorithm, that have a preference toward societies. The world wide web is considered as enormous knowledgebase repository where you can search various kinds of data and information. It is notice that, it is not necessary that TrustRank would cover every topic that exists on the web by the used of seed set coverage. Various other authors may address these issues with the effective use of the data from partition of seed set in term of computing the trust score for every single title distinctly.

In Given algorithm, set of seed, according to the data or topic, Trust Rank algorithm segregated the portion of seed set into different category. For that the equation is as follows:

$$
\left(\sum_{i=1}^{n} m_{i}\right) \times t=\sum_{i=1}^{n}\left(m_{i} \times t_{i}\right)
$$

This balance is a description of the theorem of Linearity. In this we simply assume that $\mathrm{T}$ is denoted as a seed set, which may be divided into 
no. of sub sets that is $\mathrm{n}$ shown like $\mathrm{T} 1, \mathrm{~T} 2$, T3...up to $\mathrm{Tn}$ and so on where each would contains mi Á (1 i n) seeds. $t$ in this considered as the TrustRank scores which is estimated as a seed set of $\mathrm{T}$ and $\mathrm{Ti}$ the score calculated by the Trust Rank with the set $\mathrm{Ti}$ A (1 i n) which may be represented as this. The summation shows the total no of seed which is equal to the no. of seed in partition is the product of the individual partition. so equation for transformation is

$$
t=\sum_{j=1}^{n} \frac{m_{n}}{\sum_{i=1}^{n} m_{i}} \cdot t_{n}
$$

By combining the two techniques, simple summation and quality bias is used to generate score of topical trust by showing a page with a single measure. To calculate the plain summation one add up all topic by trust scores in the Trust Rank algorithm, to generate the Topical TrustRank score. For the community into consideration the average value of the pages with seed in the Quality bias.

\section{Result and Observation}

The following number of observations is drawn from the results reported:

Comparison of Host-Rank algorithm and TrustRank algorithm has been to present effectiveness of the Trust-Rank algorithm. Compare and contrast various algorithms of link-based spam detection on web.

There are 4 derivatives of Trust-Rank were discussed such as Anti-Trust-Rank algorithm, topical Trust-Rank algorithm, Diffusion-Rank algorithm, and link-variable Trust-Rank algorithm.

Implementation of Host-Rank algorithm shown that this algorithm is more prone to spamming as compared to Trust-Rank algorithms.

In order to generate good result, it is better to join spam detection algorithms along with ranking algorithms.
With the help of the seed selection algorithm in Trust-Rank one can easily identify the additional pages. There is a scope of further exploration between dampening and splitting for trust propagation. Many of algorithms are used to set seed propagation for good seed set or bad seed set. By combining they will produce good result.

\section{Conclusion}

Try to present on a organized review of web spam detection techniques with the emphasis on algorithms and underlying principles. Categorize all existing algorithms into three categories based on the type of information they use i.e. is contentbased methods, link-based methods, and additional methods based on non-traditional data based on the user behavior with the different sessions.

The term web log gain the incremental gain in the field of web mining. Each time when small set of access sequence are added mine access from scratch. Incremental web mining has gain the advantage in this matter.

In order to make the information more useful, there is a requirement of maximal web mining that supports the information in essential manner. In proposed algorithm the frequency of user mining using techniques is focus on the consideration but attributes like no. of back access, time devoted on each page will also be considered performance enhancing.

To generate absolute ranking result it is good when we merge ranking algorithms along with spam detection algorithms. For finding out the more pages there is method called seed selection algorithm in TrustRank is a appropriate selection on the bases of outcome. There is a scope for the interaction between splitting for trust propagation and dampening between pages. Most algorithms use a good seed set or bad seed set for propagation. It is a believed that task of point out the presence of spam pages can be done effectively and efficiently with the help of both 
good seed set to propagate trust and a bad seed set to propagate trust. In addition, machine learning can be used to contribute in discovering web spam pages.

\section{References}

[1]. Abe, T., Miyake, J., Kawashima, M., \& Takahashi, K. (2005)."Spam filtering withcryptographic ad-hoc email addresses", In IEEE SAINT-W02005, pp. 114-117.

[2]. Agrawal, B., Kumar, N., \&Molle, M. (2005). "Controlling spam emails at the routers". In Proceedings of the IEEE International Conference on Communications, ICC 2005, Vol. 3, pp. 15881592.

[3]. Aharon, M., Elad, M. \&Bruckstein, A., (2006), "K-SVD: an algorithm for designing overcomplete dictionaries for sparse representation", Signal Process IEEE Trans 54(11):4311-4322.

[4]. Ahonen, T., Hadid, A., Pietikainen, M., (2006), "Face description with local binary patterns: application to face recognition",PatternAnalMachIntell IEEE Trans 28(12):20372041.

[5]. Algur, S., \&Pendari N. (2012), "Hybrid spamicity score approach to web spam detection" in Pattern Recognition, Informatics and Medical Engineering (PRIME), 2012 International Conference on. IEEE, pp. 36-40.

[6]. Amleshwaram, A., Reddy, N., Yadav, S., Gu, G., \& Yang, C. (2013.), “Cats:Characterizing automation of twitter spamme”, Technical report, Department of Electrical and Computer Engineering Texas A\&M University, College Station, TX 77843.

[7]. Androutsopoulos, I., Koutsias J., Chandrinos K. V., and Spyropoulos C. D. (2000)." AnEvaluation of Naive Bayesian AntiSpam Filtering.' In Proceedings of the Workshopon Machine Learning in the New Information Age, 11th European Conferenceon Machine Learning, edited by G. Potamias, V. Moustakis, and M. vanSomeren, 9-17. Barcelona, Spain: ECML.

[8]. Algur, S., \&Pendari N. (2012), "Hybrid spamicity score approach to web spam detection", in Pattern Recognition, Informatics and Medical Engineering (PRIME), 2012 International Conference on. IEEE, pp. 36-40. 